\section{Digestive \\ Diseases}

Allocca, M. 199

Beaugerie, L. 254

Calvo, P. 229

Carter, D. 194

Casiraghi, S. 218

D'Haens, G.R. 222

Danese, S. 199

De Vos, M. 239

Elewaut, D. 239

Eliakim, R. 194

5-Aminosalicylic acid 248

Abdominal ultrasound 199

Ankylosing spondylitis 239

Anti-TNF therapy 207

Azathioprine 233

Biologics 254

Bowel resection 218

Cancer recurrence 254

Chemoprevention 248

Chromoendoscopy 244

Colonic biopsies 244

Colorectal cancer 244, 248

Computed tomography enterography 199

Crohn's disease 194, 199, 202, 207, 218, 222,229

Device-assisted enteroscopy 194

Diagnosis 202

Disease course 186
Feagan, B.G. 213

Fiocchi, C. 186

Fiorino, G. 199

Foschi, D. 218

Gerner, R. 233

Griffiths, A.M. 202

Khanna, R. 213

Lopez, A. 248

Louis, E. 207

Moschen, A.R. 233

Mosli, M.H. 213
Pablo, L. 229

Pellisé, M. 244

Peyrin-Biroulet, L. 248

Rieder, F. 186

Sampietro, G.M. 218

Tilg, H. 233

van Lent, A.U. 222

Van Praet, L. 239

Wieser, V. 233

\title{
Subject Index Vol. 31, No. 2, 2013
}

Evidence-based medicine 213

Fibrosis 186

Fistula 186, 218

Hepatotoxicity 233

Histology 202

Immunosuppressants 207

Immunosuppressive therapy 254

Inflammatory bowel disease 186,213 ,

$$
\text { 244, } 247
$$

- $\quad$ diseases 239

Magnetic resonance imaging enterography 199

Non-alcoholic fatty liver disease 233

Ocular complications 229

Osteoarticular manifestations 239
Postoperative recurrence 222

Primary sclerosing cholangitis 233

Randomized trials 213

Second cancer 254

Small bowel capsule endoscopy 194

Spondyloarthritis 239

Steroids 229

Strictureplasty 218

Surgery 218, 222

The Lémann score 199

Treatment 222

Ulcerative colitis 207, 229 